\title{
Search for dark matter signatures in the cosmic-ray electron and positron spectrum measured by the Fermi Large Area Telescope
}

\author{
M. N. Mazziotta*1, A. Cuoco ${ }^{2,3}$, P. De La Torre Luque ${ }^{1,4}$, F. Loparco ${ }^{1,4}$ and D. Serini ${ }^{1,4}$ \\ on behalf of the Fermi LAT collaboration ${ }^{\dagger}$ \\ ${ }^{1}$ Istituto Nazionale di Fisica Nucleare - Sezione di Bari, I-70126 Bari, Italy \\ ${ }^{2}$ RWTH Aachen University, Institute for Theoretical Particle Physics and Cosmology (TTK), \\ D-52056 Aachen, Germany \\ ${ }^{3}$ CNRS - Laboratoire d'Annecy de Physique des Particules, F-74940 Annecy, France \\ ${ }^{4}$ Dipartimento Interateneo di Fisica dell'Università e del Politecnico di Bari, I-70125 Bari, Italy \\ E-mail: marionicola.mazziotta@ba.infn.it
}

\begin{abstract}
Over its ten years of mission the Large Area Telescope onboard the Fermi Gamma-ray Space Telescope has collected the largest ever sample of high-energy cosmic-ray electron and positron events. Possible features in their energy spectrum could be a signature of the presence of nearby astrophysical sources or of more exotic sources, such as annihilating or decaying dark matter particles in the Galaxy. In addition, the capture and subsequent annihilation of dark matter particles in the Sun via long-lived dark mediators can yield high-energy electrons and positrons reaching the Earth, which are a probe for the dark matter-nucleon scattering cross section. We present the results of the search for possible features in the cosmic-ray electron and positron spectrum from our Galaxy and towards the Sun. We set constraints on the the velocity-averaged dark matter annihilation cross section and on the dark matter-nucleon scattering cross section up to dark matter masses of $1.7 \mathrm{TeV}$.
\end{abstract}

36th International Cosmic Ray Conference -ICRC2019-

July 24th - August 1st, 2019

Madison, WI, U.S.A.

\footnotetext{
${ }^{*}$ Speaker.

${ }^{\dagger}$ The Fermi LAT Collaboration acknowledges generous ongoing support from a number of agencies and institutes that have supported both the development and the operation of the LAT as well as scientific data analysis. These include the National Aeronautics and Space Administration and the Department of Energy in the United States, the Commissariatà l'Energie Atomique and the Centre National de la Recherche Scientifique / Institut National de Physique Nucléaire et de Physique des Particules in France, the Agenzia Spaziale Italiana and the Istituto Nazionale di Fisica Nucleare in Italy, the Ministry of Education, Culture, Sports, Science and Technology (MEXT), High Energy Accelerator Research Organization (KEK) and Japan Aerospace Exploration Agency (JAXA) in Japan, and the K. A. Wallenberg Foundation, the Swedish Research Council and the Swedish National Space Board in Sweden. Additional support for science analysis during the operations phase is gratefully acknowledged from the Istituto Nazionale di Astrofisica in Italy and the Centre National d'Études Spatiales in France. This work performed in part under DOE Contract DE-AC0276 SF00515.
} 


\section{Introduction}

High-energy cosmic-ray electrons and positrons (herafter CREs) lose their energy mainly through synchrotron radiation and inverse Compton interactions with the low-energy photons of the interstellar radiation field during their journey in the Galaxy. Therefore, CREs reaching the Earth with energies above $100 \mathrm{GeV}$ should be produced by a few nearby sources [1,2]. An alternative production mechanism for high-energy CREs could arise due to the annihilation or decay of dark matter (DM). In this case, the CRE energy spectrum is expected to exhibit a cut-off at the energy corresponding to the DM mass [3]. Therefore, the signature of a DM contribution to the CRE spectrum would be an "edge"-like feature at energies close to the DM mass.

DM particles from the Galactic halo passing through the Sun are expected to scatter off nuclei, lose energy, and be trapped by the solar gravitational field. Multiple scatterings cause DM to accumulate in the centre of the Sun. If DM particles can self-annihilate, the capture is then balanced by the annihilation, leading to the equilibrium between the two rates. DM annihilations are expected to yield Standard Model (SM) particles that, with the exception of neutrinos, will be absorbed by the Sun. However alternative models have been proposed, which predict the possibility of DM to annihilate into long-lived mediators that can escape the Sun and then decay into SM particles. In a further class of models, DM scatters inelastically off Sun nuclei, leading to a significant accumulation of DM particles just outside the surface of the Sun. Also in this case the DM annihilations could produce SM particles which reach the Earth. In the framework of these models, electrons and positrons produced from DM captured by the Sun can be detectable at Earth, via either decay of the mediator or direct DM annihilation [4] (see also [5] and reference therein).

In this work, we performed a comprehensive analysis of the CRE data [6] collected by the Fermi Large Area Telescope (LAT) [7] to search for possible features in the spectrum originating either from the direct annihilation of DM particles in the Galactic halo into $e^{+} e^{-}$pairs [8] or towards the Sun, where we searched for either delta-like lines or box-like spectral edges [9].

\section{CREs originating from dark matter}

\subsection{Spectrum of cosmic-ray electrons and positrons from DM annihilations in the halo}

The spectrum of CREs produced from DM annihilations in the Galaxy has been evaluated with a customized version of the propagation code DRAGON [10,11], in which the cross sections for the production of secondary particles are taken from Ref. [12]. We set the propagation model of CRs in the Galaxy assuming a source term distribution, a gas density distribution and the interstellar radiation field (ISRF) and Galactic magnetic field model (GMF). We assume that the scalar diffusion coefficient depends on the particle rigidity $R$ and on the distance from the Galactic plane $z$ according to the parameterization $D=D_{0} \beta^{\eta}\left(R / R_{0}\right)^{\alpha} e^{|z| / z_{t}}$ [8]. We set $\alpha=0.33$ according to the recent boron to carbon ratio (B/C) from the AMS-02 data [13], $R_{0}=4 \mathrm{GV}$ and $z_{t}=4 \mathrm{kpc}$, while $D_{0}$ and $\eta$ are tuned to the B/C AMS-02 data, also setting the nuclei injection spectra to reproduce the VOYAGER 1 data at low energy [14]. We have found that the $\mathrm{B} / \mathrm{C}$ data are reproduced setting $D_{0}=7.4 \times 10^{28} \mathrm{~cm}^{2} \mathrm{~s}^{-1}$ and $\eta=-0.1$. A reacceleration model is also adopted to reproduce the $\mathrm{B} / \mathrm{C}$ data at low energy, setting the Alfvén velocity to $v_{\mathrm{A}}=52 \mathrm{~km} \mathrm{~s}^{-1}$. 
With this propagation model in hand, we have evaluated the spectra of CREs at Earth produced by DM annihilations in our Galaxy still using the DRAGON code. We assume a NavarroFrenk-White DM density profile [15] with a local density $\rho_{\odot}=0.41 \mathrm{GeV} \mathrm{cm}^{-3}$ [16]. The inclusive yields of $e^{ \pm}$from DM annihilations are taken from [3], including electroweak corrections [17]. Fig. 1 shows, for each DM mass, the expected CRE spectra at Earth calculated assuming a velocity-averaged annihilation cross section $\langle\sigma \mathrm{v}\rangle=3 \times 10^{-25} \mathrm{~cm}^{3} \mathrm{~s}^{-1}$ and compared with the Fermi LAT [6], AMS02 [18], CALET [19] and DAMPE [20] data. The spectra scale lineary with $\langle\sigma \mathrm{v}\rangle$ and $\rho_{\odot}^{2}$. The CRE spectra from DM annihilations are very hard and exhibit a cutoff at the energy corresponding to the mass of the DM particle, which is referred to as an "edge-like" feature.

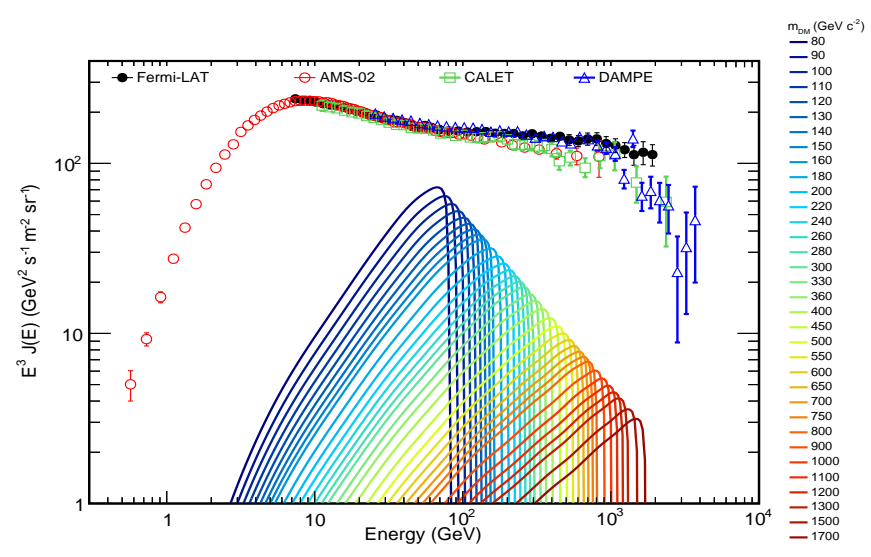

Figure 1: Galactic CRE spectra at Earth. The expected contributions from possible DM annihilations in the Galaxy (corresponding to $\langle\sigma \mathrm{v}\rangle=3 \times 10^{-25} \mathrm{~cm}^{3} \mathrm{~s}^{-1}$ ) are compared with the data from the Fermi LAT [6], AMS-02 [18], CALET [19] and DAMPE [20].

\subsection{Dark Matter models from the Sun and electron-positron energy spectrum}

We assume two scenarios for the production of CREs due to annihilations of DM particles captured inside the Sun: i) capture via elastic scattering and subsequent annihilation in the core of the Sun into $e^{+} e^{-}$pairs through a light intermediate state $\phi$; ii) capture via inelastic scattering and subsequent annihilation directly into $e^{+} e^{-}$pairs outside the Sun.

In the first model, DM particles are captured by the Sun through elastic scatterings, lose energy through subsequent scatterings, and eventually thermalize and sink to the core, where they annihilate. When capture and annihilation reach equilibrium, the annihilation rate is given by:

$$
\Gamma_{\text {ann }}=\frac{1}{2} \Gamma_{\text {cap }}=\frac{1}{2} C_{\text {ann }} N_{\chi}^{2}
$$

We assume that DM particles annihilate into a light intermediate state $\phi$, i.e., $\chi \chi \rightarrow \phi \phi$, with the $\phi$ subsequently decaying to standard model particles. The $\phi$ are assumed to be able to escape the Sun without further interactions, with each $\phi$ decaying to an $e^{ \pm}$pair. If the decay happens outside the surface of the Sun, the $e^{ \pm}$can reach the Earth and may be detectable in the form of an excess of CREs from the direction of the Sun. The DM particles are assumed to annihilate at rest in the core of the Sun. Therefore, in the lab frame, the energy of the $\phi$ will be equal to the mass of 
the DM particle, i.e. $E_{\phi}=m_{\chi}$. We assume $\phi$ to be a light scalar such that $m_{\phi} \ll m_{\chi}$. Under this assumption, the $\phi$ are relativistic, i.e. $\gamma_{\phi}=E_{\phi} / m_{\phi} \gg 1$. The angular dispersion of the $e^{ \pm}$pair with respect to the direction of the parent $\phi$ is of the order of $1 / \gamma_{\phi} \sim 0$, and therefore the $e^{ \pm}$will keep the same direction as the $\phi$.

Indicating with $L$ the $\phi$ decay length, the flux of $e^{ \pm}$produced from $\phi$ s decaying and detected at the Earth is given by:

$$
\Phi_{\mathrm{DM}}(E)=N(E) \frac{\Gamma_{\text {cap }}}{4 \pi D^{2}}\left(e^{-R_{\odot} / L}-e^{-D / L}\right)
$$

where $R_{\odot}$ is the Sun radius and $D$ is distance of the Sun center to the Earth. The DM $e^{ \pm}$spectrum in this scenario acquires a box-like shape centered on $E=m_{\chi} / 2$, with a width depending on the mediator mass $m_{\phi}$. In the limit $m_{\phi} \ll m_{\chi}$, the dependence on $m_{\phi}$ disappears and the box extends from $E=0$ to $E=m_{\chi}$. We can therefore write $N(E)=2 H\left(m_{\chi}-E\right) / m_{\chi}$, with the factor 2 accounting for the $e^{ \pm}$multiplicity (2) for each mediator and $H$ the Heaviside step function. We assume that mediators can pass through Sun without attenuation. The capture rate has been evaluated with the DARKSUSY code version $6.1 .0[21,22]$ assuming the default settings, with a local DM density $\rho_{\odot}=0.3 \mathrm{GeV} / \mathrm{cm}^{3}$, a Maxwellian velocity distribution with average velocity $v_{\odot}=220 \mathrm{~km} / \mathrm{s}$ and velocity dispersion $v_{r m s}=270 \mathrm{~km} / \mathrm{s}$, and setting the DM-nucleon cross section to $\sigma=10^{-40} \mathrm{~cm}^{2}$ (in both spin independent and spin dependent cases). The capture rate scales linearly with both $\sigma$ and the local DM density $\rho_{\odot}$.

In the second model, a DM particle $\chi$ that scatters inelastically off a solar nucleon $N$ will produce an excited state $\chi^{*}$ with a slightly heavier mass, i.e. $\chi+N \rightarrow \chi^{*}+N$. To undergo a scattering, the DM particle needs an energy $E \geq \Delta\left(1+m_{\chi} / m_{N}\right)$, where $\Delta=m_{\chi}^{*}-m_{\chi}$ is the mass splitting parameter. The captured particles will remain on relatively large orbits. As a result, the density of captured DM particles outside the Sun may not be negligible, and the annihilation of those particles to $e^{ \pm}$could thus produce an observable flux of CREs from the direction of the Sun. Assuming that DM particles annihilate at rest and that CREs do not lose energy when travelling from the Sun to the Earth, their spectrum will be a line at $E_{e^{ \pm}}=m_{\chi}$. We also assume that all annihilations occur close to the surface of the Sun, since the density of DM rapidly decreases with the distance from the Sun. As for the previous case of elastic scattering, we assume equilibrium, i.e. $\Gamma_{\text {ann }}=\frac{1}{2} \Gamma_{\text {cap }}$. Defining $f_{\text {out }}$ as the fraction of DM annihilations occuring outside the Sun at a given time, we can write the following equation:

$$
\Gamma_{\text {ann,out }}=f_{\text {out }} \Gamma_{\text {ann }}=\frac{1}{2} f_{\text {out }} \Gamma_{\text {cap }}
$$

We use the capture rate $\Gamma_{\text {cap }}$ as a function of DM mass $m_{\chi}$ and of mass splitting $\Delta$ as given in Fig. 2 of Ref. [23], and adjust the overall capture rate with $\sigma_{0}$.

We use the parameter $f_{\text {out }}$ as a function of the splitting mass parameter $\Delta$ as calculated by Ref. [4] for the DM captured by the Sun via inelastic scattering and $m_{\chi}=1 \mathrm{TeV}$. We assume that the dependence on $m_{\chi}$ is weak for the mass range of the present work, also following the prescriptions of Ref. [4]. We note that the uncertainties in the calculation of $f_{\text {out }}$ are large, and a detailed study is beyond the scope of this paper. However, the present results can be easily rescaled. The flux of $e^{ \pm}$at the Earth due to the DM annihilations captured nearby the Sun via inelastic scattering is 


$$
\Phi_{D M}(E)=2 \frac{\Gamma_{a n n, o u t}}{4 \pi D^{2}} \delta\left(E-m_{\chi}\right)
$$

where the factor 2 accounts that 2 CREs are emitted per annihilation of each pair of DM particles. However, we assume that only one particle of the $e^{+} e^{-}$pair emitted per annihilation nearby the Sun surface can reach the Earth, so we assume that the flux of CRE observed is a factor 2 smaller than that given by Eq. 2.4, and this would result in conservative limits.

\section{Analysis Method}

For the present work we analyzed the same dataset used in Refs. [6] and [8]. Full details on the event selection are given in Ref. [6]. We have implemented a fitting procedure in sliding energy windows to search for possible local peaks (either bumps, box or lines) on the top of a smooth CRE spectrum. In each energy window we model the CRE intensity as $I(E)=I_{0}(E)+I_{f}(E)$, where $I_{0}(E)$ is the "smooth" part of the spectrum and $I_{f}(E)$ describes the possible feature. Since the energy windows are narrow, we assume that the smooth part of the spectrum can be described by a power-law (PL) model $I_{0}(E)=k\left(E / E_{0}\right)^{-\gamma}$, where $\gamma$ is the PL spectral index and the prefactor $k$ corresponds the CRE intensity at the scale energy $E_{0}$, fixed to $1 \mathrm{GeV}$.

In our analysis, we assume three models for $I_{f}(E)$ : (i) a delta-like (hereafter line model) feature, $I_{f}(E)=s \delta\left(E_{w}-E\right)$, where $s$ represents the intensity of the line in units of $\mathrm{m}^{-2} \mathrm{~s}^{-1} \mathrm{sr}^{-1}$; (ii) a box-like (hereafter box model) feature, $I_{f}(E)=s H\left(E_{w}-E\right)$, where $s$ represents the intensity of the box in units of $\mathrm{GeV}^{-1} \mathrm{~m}^{-2} \mathrm{~s}^{-1} \mathrm{sr}^{-1}$; (iii) a spectrum produced by DM annihilating into CREs, $I_{f}(E)=s I_{D M}\left(E \mid m_{D M},\langle\sigma \mathrm{v}\rangle, \ldots\right)$, where $I_{D M}(E)$ is the intensity of CREs from DM observed near the Earth, which is calculated in Sec. 2.1, and the parameter $s$ represents the scale of the annihilation cross-section implemented in the model ( $s$ corresponds to $\langle\sigma \mathrm{v}\rangle$ in units of $3 \times 10^{-26} \mathrm{~cm}^{3} \mathrm{~s}^{-1}$ ). Here we indicate with $\delta$ the Dirac delta function and with $H$ the Heaviside step function, while $E_{w}$ is the energy corresponding to the center of the sliding window. The line model will be used in the analysis of CREs from the Sun (a spectral line is expected from direct annihilations of DM into $e^{ \pm}$ pairs, see Sec.2.2), but also in the analysis of CREs from the Galactic Halo.

Starting from the model, we can calculate the expected counts in each CRE observed energy bin $E_{j}$ as:

$$
\mu_{j}=\mu\left(E_{j}\right)=t \int d E \mathscr{R}\left(E_{j} \mid E\right) I(E)
$$

where $E$ is true (Monte Carlo) energy, $\mathscr{R}\left(E_{j} \mid E\right)$ is the instrument response matrix (acceptance) which incorporates the energy resolution of the LAT, and $t$ is the integrated livetime. For our fitting procedure we define a $\chi^{2}$ function as follows:

$$
\chi^{2}=\sum_{j=1}^{N} \frac{\left(n_{j}-\mu_{j}\right)^{2}}{n_{j}+f_{s y s t}^{2} n_{j}^{2}}
$$

where $N$ is the number of energy bins used for the fit. The denominator of each term in the summation includes the sum in quadrature of statistical Poisson fluctuations $\left(\sqrt{n_{j}}\right)$ and systematic uncertainties $\left(f_{\text {syst }} n_{j}\right)$. The analysis of the Galactic Halo is performed looking at CREs coming from the whole sky. On the other hand, in the case of the Sun analysis, we define a signal region 
and a background control region as cones with fixed angular radius and axis pointing towards the Sun and the anti-Sun respectively, and we fit simultaneously the count distributions from the two regions. To estimate the parameters $\{k, \alpha, s\}$ which minimize the $\chi^{2}$ we use the MINUIT code within the ROOT toolkit; the values of the parameters at a 95\% confidence limit (CL) are evaluated using MINOS and setting the error confidence level to 2.71 .

We have performed our fits scanning an energy range extending from $42 \mathrm{GeV}$ to about $2 \mathrm{TeV}$. This interval has been divided in 64 bins per decade, equally spaced on a logarithmic scale. We perform the fit in sliding energy windows with a half-width of $0.35 E_{w}$, that well contain the possible features. In fact, the LAT energy resolution for the CRE selection at 95\% containment ranges from about $15 \%$ at $42 \mathrm{GeV}$ to about $20 \%$ at $1 \mathrm{TeV}$ and increases up to $35 \%$ at $2 \mathrm{TeV}$, as shown in Ref. [6]. On the other hand, when searching for a DM signal from direct annihilation to $e^{ \pm}$pairs in the Galactic Halo, we have selected fit windows centered on the candidate DM mass $m_{D M}$ with a half-width of $0.5 m_{D M}$. We have also tested different energy binnings and different window sizes yielding comparable results [8].

We evaluate the expectation bands for our results, i.e. the sensitivity to the null hypothesis, using a pseudo-experiment technique. As a starting point, we fit the observed CRE count distribution with a simple PL model in the whole energy range, and we use this model as a template to evaluate the expected counts in each energy bin.

\section{Results and Conclusions}

The current analysis yields no evidence for any feature like a line, a box or a possible DM signal from the Galactic Halo. We set constraints on the line intensity and on the velocity-averaged DM annihilation cross section in the case of galactic CREs, and limits on the DM cross section per nucleon in the case of solar CREs. Our limits extend up to DM masses of $1.7 \mathrm{TeV} / \mathrm{c}^{2}$

Fig. 2 shows the upper limits at $95 \%$ confidence level on the parameters describing the feature ( $s$ for the line, $\langle\sigma \mathrm{v}\rangle$ for the DM signal) from the analysis of the Galactic Halo. In the right panel of Fig. 2 we also include dashed-dotted and dotted black lines showing variations of the limits on $\langle\sigma \mathrm{v}\rangle$ assuming that $\rho_{\odot}$ can vary in the range $(0.25-0.7) \mathrm{GeV} \mathrm{cm}^{-3}$ and that the ISRF together with the GMF can vary by $\pm 50 \%$, respectively. The green and yellow bands show the $68 \%$ and $95 \%$ CL expectation bands, respectively, calculated from the pseudo-experiments. Since the limits lie within the 95\% CL expectation bands, the Fermi LAT data do not provide evidence of any feature at the $2 \sigma$ (local) level, either in the case of a delta-like line or in the case of a signal from DM annihilations in the Galaxy.

CREs originating from DM captured in the Sun should yield a signal peaked towards the direction of the Sun with an angular extension determined by the heliospheric magnetic field. A detailed study of the effects of the heliomagnetic field is beyond the goal of the present paper, and in general is not straightforward because the geometry of the field is rather complex. Therefore, to take into account the effect of heliomagnetic field, in the present analysis we have chosen as signal region a cone with aperture of $30^{\circ}$ pointing towards the Sun (see Ref. [9] for alternative RoIs).

The left panel of Fig. 3 shows the constraints on DM annihilation to $e^{+} e^{-}$via an intermediate state, assuming that the capture of DM takes place either via spin independent (black lines) or spin dependent (red lines) elastic scattering. The constraints have been calculated for four values of the 

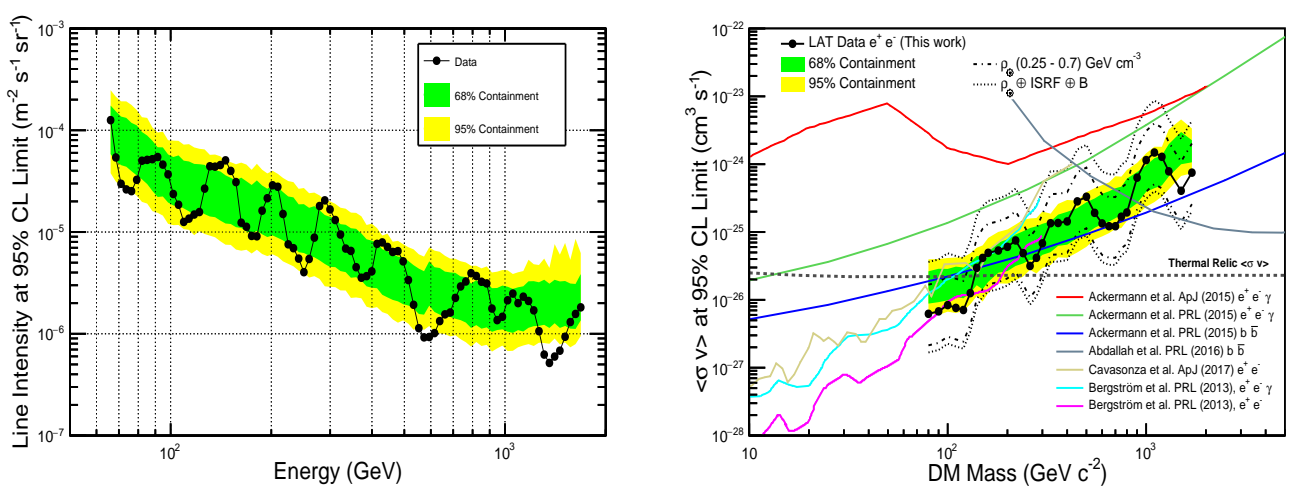

Figure 2: Upper limits on the galactic CRE spectral features. The left plot has been obtained assuming a delta-like line feature on the top of a power-law spectrum; the right plot has been obtained assuming a feature due to DM annihilating into CREs on the top of a power-law spectrum. The plots show the upper limits at $95 \% \mathrm{CL}$ on the parameter describing the feature ( $s$ for the line, $\langle\sigma \mathrm{v}\rangle$ for the DM signal). The dashed-dotted and the dotted black lines in the right plot indicate the variations of the limits on $\langle\sigma \mathrm{v}\rangle$ for reasonable variations of the local DM density, of the ISRF and of the GMF (see discussion in the text). The dashed line in the right plot indicates the thermal relic cross section from Steigman et al. [24]. The colored lines indicate the upper limits on $\langle\sigma v\rangle$ taken from Refs. [25-29].
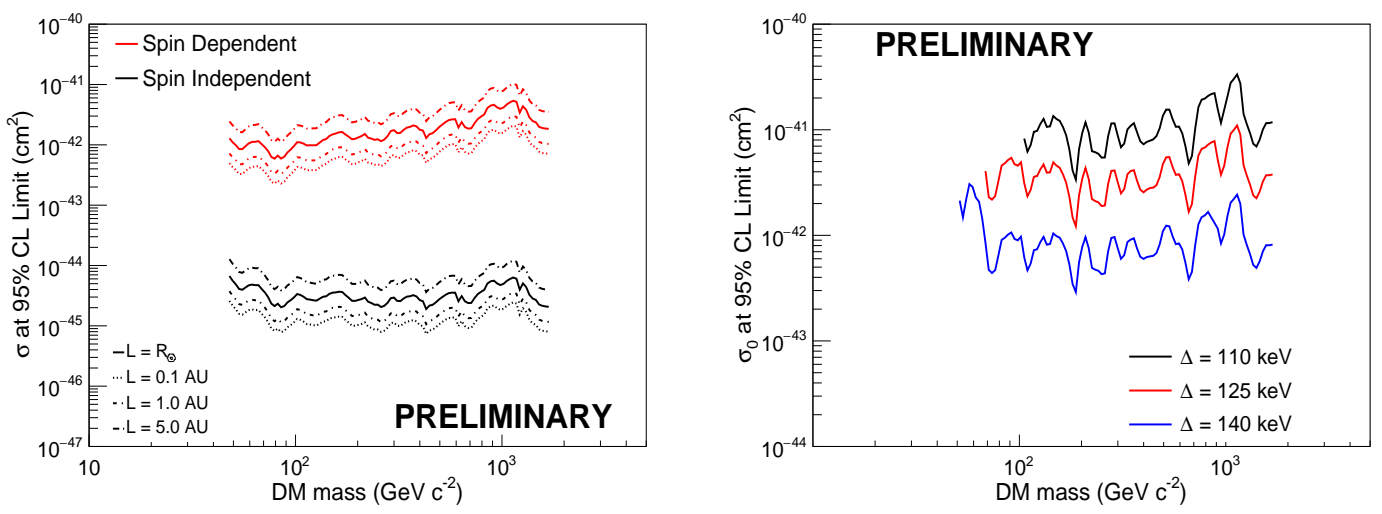

Figure 3: Results of the search features in a $30^{\circ}$ RoI towards to the Sun in the case of the box (left panel) and line models (right panel). The left plot shows the upper limits on the elastic DM-nucleon cross section for both the spin dependent and spin independent cases, for four different decay lengths of the mediator $L=R_{\odot}$, $0.1,1$ and $5 \mathrm{AU}$; the right plot shows the upper limits on the cross section per nucleon $\sigma_{0}$ in the inelastic case for DM annihilation into $e^{+} e^{-}$as a function of energy, calculated for three values of the splitting mass parameter, i.e., $\Delta=110,125$ and $140 \mathrm{keV}$ (right panel).

decay length of the intermediate state, $L=R_{\odot}, 0.1,1$ and 5 AU. The right panel of Fig. 3 shows the limits on the cross section per nucleon $\sigma_{0}$ for DM capture via inelastic scattering with subsequent direct annihilation into $e^{+} e^{-}$. These limits have been calculated for three values of the splitting mass parameter, $\Delta=110,125$ and $140 \mathrm{keV}$.

In the current analysis we constrain CRE signals produced by DM from the Galactic Halo and from the Sun covering the mass range from $42 \mathrm{GeV}$ to $1.7 \mathrm{GeV}$. A similar analysis towards the Sun has been also performed with photons down to energies of a few $\mathrm{GeV}$, also presented in this 
conference [30].

\section{References}

[1] M. Ackermann et al. [Fermi-LAT Collaboration], Phys. Rev. D 82, 092003 (2010) [arXiv:1008.5119].

[2] S. Abdollahi et al. [Fermi-LAT Collaboration], Phys. Rev. Lett. 118091103 (2017)

[3] M. Cirelli et al., JCAP 1103, 051 (2011) Erratum: [JCAP 1210, E01 (2012)] [arXiv:1012.4515].

[4] P. Schuster et al., Phys. Rev. D 82 115012, 2010.

[5] M. Ajello et al. [Fermi-LAT Collaboration] Phys. Rev. D, 84 032007, 2011.

[6] S. Abdollahi et al. [Fermi-LAT Collaboration] Phys. Rev. D, 95 082007, 2017.

[7] W. B. Atwood et al. [Fermi-LAT Collaboration] Astrophys. J., 697 1071, 2009.

[8] M. N. Mazziotta et al. Phys. Rev. D 98022006 (2018) [arXiv:1712.07005].

[9] A. Cuoco, P. De La Torre Luque, F. Gargano, M. Gustafsson, F. Loparco, M. N. Mazziotta and D. Serini [Fermi-LAT Collaboration], A search for dark matter cosmic-ray electrons and positrons from the Sun with the Fermi Large Area Telescope To be submitted

[10] C. Evoli, D. Gaggero, D. Grasso and L. Maccione, JCAP, 0810 018, 2008.

[11] D. Gaggero, L. Maccione, G. Di Bernardo et al., Phys. Rev. Lett., 111 021102, 2013.

[12] M. N. Mazziotta at al., Astropart. Phys., 81 21, 2016.

[13] M. Aguilar et al., Phys. Rev. Lett., 117 231102, 2016.

[14] E. C. Stone et al., Science, 341 150, 2013.

[15] J. F. Navarro, C. S. Frenk, and S. D. M. White, Astrophys. J., 462 563, 1996.

[16] P. Salucci, F. Nesti, G. Gentile, and C. F. Martins, Astron. Astrophys., 523 A83, 2010.

[17] P. Ciafaloni et al., JCAP, $\mathbf{0 3} 019,2011$.

[18] M. Aguilar et al., Phys. Rev. Lett., 113 221102, 2014.

[19] O. Adriani et al., Phys. Rev. Lett., 119 181101, 2017.

[20] G. Ambrosi et al., Nature, 552 63, 2017.

[21] P. Gondolo et al., JCAP, 0407 008, 2004.

[22] T. Bringmann et al., JCAP, 1807 033, 2018. http://www. darksusy.org/

[23] A. Menon, R. Morris, A. Pierce, and N. Weiner, Phys. Rev. D, 82 015011, 2010.

[24] G. Steigman, B. Dasgupta, and J. F. Beacom, Phys. Rev. D, 86 023506, 2012.

[25] M. Ackermann et al. [Fermi-LAT Collaboration], Phys. Rev. Lett., 115 231301, 2015.

[26] M. Ackermann et al. [Fermi-LAT Collaboration], Astrophys. J., 812 159, 2015.

[27] L. Bergstrom et al., Phys. Rev. Lett., 111 171101, 2013.

[28] L. A. Cavasonza, H. Gast, M. Kramer, M. Pellen, and S. Schael, Astrophys. J., 83936, 2017.

[29] H. Abdallah et al., Phys. Rev. Lett., 117 111301, 2016.

[30] D. Serini, F. Loparco and M. N. Mazziotta, Constraints on DM scattering with long-lived mediators using gamma-rays from the Sun In proceeding of 36th ICRC POS ( ICRC2019) 5442019 